ISBN 978-981-14-1455-8

Proceedings of 2019 the 9th International Workshop on Computer Science and Engineering

WCSE_2019_SPRING

Yangon, Myanmar, February 27-March 1, 2019, pp. 107-111

doi:10.18178/wcse.2019.03.019

\title{
Trust Recommendation System in Online Shopping Using Text Mining
}

\author{
Hla Sann Sint ${ }^{+}$and Khine Khine Oo \\ University of Computer Studies, Myanmar
}

\begin{abstract}
In online society, understanding the methods of trust is essential. In addition, issues of trust are necessary to several solving problems corporate responsibility, online shopping, and the social system. Trust is a concept with many facets and dimensions. Many trust evaluations have been proposed, which used direct ratings from user to calculation or propagation of trust values. In some web-based social networks technology that is no direct rating value where users are calcu lated by binary relationships. The benefit of th is paper is to examine the importance of trust in online shopping, which trust value is calculated by without any direct ratings from consumers. In this calculation, which is based calculate on opinion, user similarity is based from the texts comments by the users for the trust with text-mining techniques using Sentiment Analysis and then evaluate with Improved PageRank. Moreover, this system approach in web-based social networks and proposed technique can also be help in direct rating methods to calculate the correct values of trust in online services.
\end{abstract}

Keywords: Online Shopping, Sentiment Analysis, Improved PageRank Algorithm, Text Mining.

\section{Introduction}

In today's technology, communication has very grown and very easy and cheap to communicate and connect with each other. Communication techniques have improved from wired devices to wire less. The internet has also fast changes in the forms of communications that are available and currently being used. It has led the rise of value of social networks. Trust can be very valuable in social interactions .In society, trust needs for not only people, but also institutions and systems. There is a general consensus among contemporary social system that social trust is important, for both social and political reasons. One important issue in online services is online consumers are luck of faith to shop online is because of the fundamental lack of believe to trust, which currently exists between business and customers in online social networks.

In this system, sentiment of users regarding the online services is considered. The opinions of the consumers are implied by comments, likes and shares. The quality of delivery products is really good or not and price is fair or not that depends on user's comments, which is the important information to decide the product's quality. Moreover, product manufacturers can obtain improvement products given opinion by users. And then using Improve PageRank Algorithm can be evaluated to easy find for more trusted path.

The remainder of this paper is organized as follows: describe trust in online shopping in section 2 and related works in section 3 . Then discuss evaluation for user trust prediction in section 4 . At last, section 5 describe conclusion and future work.

\section{Trust in Online Shopping}

Online shopping attracts people because it has many advantages. Nowadays, more and more people don't have that much free time to go shopping they're busy working, studying and doing other important things. Many people choose shopping online also because they can't stand that crowded and noisy

\footnotetext{
${ }^{+}$Corresponding author.

E-mail address: hlasannsint @ gmail.com
} 
environment and spend several minutes to get what they want without stepping out of the room or standing in line for checking. On one hand, shopping online is not always a perfect to trust, it also has disadvantages. It seems easy and quick, but there's always a trap online. Other people may worry about shopping online because they fear their credit card information will be compromised. Another reason some consumers avoid shopping online is the fact that they worry that the products they purchase are not accurately portrayed in the website's picture. Therefore online shopping is offering convenience as well as disadvantages. On one hand, customers enjoy shopping without going out. On the other hand, customers need to be trust that is very important when choosing and trading online.

\section{Related Works}

Several trust computing methods have been proposed from different perspectives.

Amel Ziani, Nabiha Azizi, Didier Schwab, Monther Aldwairi, Nassira Chekkai, Djamel Zenakhra, Soraya Cheriguene[1] proposed a multilingual recommender system based on sentiment analysis using online product reviews. This paper was used semi supervised SVM for the opinions classification task to avoid the lack of annotated data problem. The experimental results suggested very high precision and a recall of $100 \%$. This results analys is evaluation provides interesting findings on the impact of integrating sentiment analysis into a recommendation technique based on collaborative filtering.

$\mathrm{Au}$ Yau Leung, Rex[2] investigated trust level online-shopping in Hong Kong. This system result based on primary data analysis and descriptive statistics, reliability analys is and regression analys is. Data collected from the scholars and students were based on by using paper-form questionnaire. The result showed factors that contribute to trust, purchase intentions, and other influencing variables must be measured.

Dimah A lahmadi and Xaio-Jun Zeng[3] proposed a framework of implicit social trust and sentiment (ISTS) based on recommendation systems from friends' short posts in microblogging as micro-reviews by using machine learning methods inc luding Naive Bayes, Logistic Regression and Decision Trees. The results show that the these short and inconsistent posts can empower the users preferences data in particular when no preferences of history were available.

R. Lydia Priyadharsini, M. Lovelin Ponn Felciah[4] proposed recommendation system based on hybrid recommendation algorithm for E-commerce web sites and the customer can easily search the products ratings, reviews are analyzed by sentiments with the help of review based MAC filtering that can be avoided fake reviews. The advantage of this system is the visual organization of the data based on the underlying structure, and a significant reduction in the size of the search space per result output.

Sonja Grabner-Kräuter[5] developed a theoretical framework, which facilitates a multi-level and multidimensional analysis of research problems related to trust in OSNs. This paper discussed different types and sources of trust from the trust literature and their importance for trust-related decisions and behaviors in OSNs. The analysis revealed social capital can be viewed both as an outcome gained by individuals in an OSN and as a tool for facilitating the governance of such spaces and also it was shown the relationship between social capital and trust is not unidirectional but reciprocal.

Some problem drawbacks have not been solved by above mentioned methods in online shopping. Most of research approaches require rating from users to calculate trust evaluations to work properly. In this paper, trust values evaluation without any user rating that can be calculated user similarity us ing sentiment analysis. These are calculated based on posts and the comments shared by the user.

\section{Evaluation for Trust Prediction}

In this section, described the procedure of evaluation trust prediction, and then the calculation with Improved PageRank method to produce the correctness trust value. In Fig. 1 describes the calculation of trust decision making.

\subsection{Collecting Information}

As explain before, the data is collected from the posts shared by users, and the texts annotated as comments. The information collected from each of these sources form a text file which would be mined to extract similarity.

\subsection{Removing Stop Words}

A stop word is a commonly used word (such "a" and "the") that a search engine has been programmed to ignore, both when indexing entries for searching and when retrieving them as the result of a search query. 
The list of words that are not to be added is called a stop list. Stop words are deemed irrelevant for searching purposes because they occur frequently in the language for which the indexing engine has been tuned.

\subsection{Replace Words}

A replace words is a convert to one word from given text. The words are converted as a single word as they are the generally similar. For example, "not too good" sentence replaces with "bad", "not bad "sentence replaces with "good", "not too bad" sentence replaces with "nice".

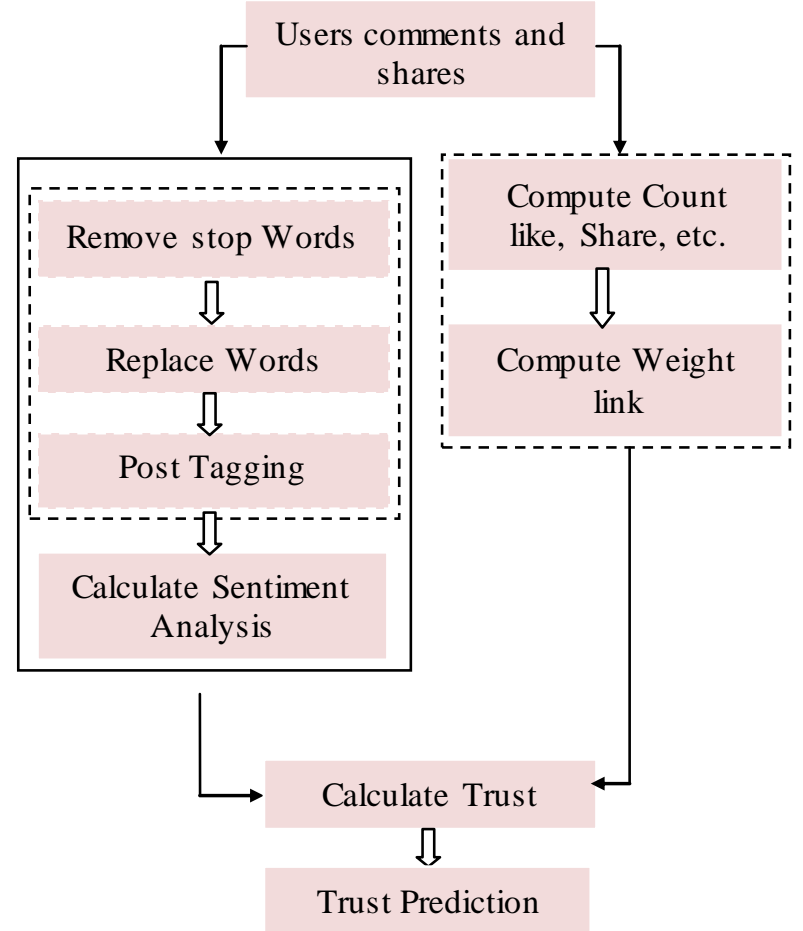

Fig.1 Evaluation for Trust Prediction Architecture

\subsection{Post Tagging}

The process of assigning a tag to a word in a corpus is Tagging. Parts of speech (POS), word classes, morpho-logical classes, or lexical tags give information based on both its definition and its context. POS Tagging is relationship with adjacent and related words in a phrase, sentence, or paragraph. There are eight basic POS have been distinguished: Noun, verb, pronoun, preposition, adverb, conjunction, adjective, and article

\subsection{Sentiment Analys is}

Sentiment analysis is the automated process of understanding an opinion mining for a given subject from written or spoken language. In this paper, can analyze how many trusts on products from online shopping calculate using comments that extract keywords and matched with database and calculate without use of any rating.

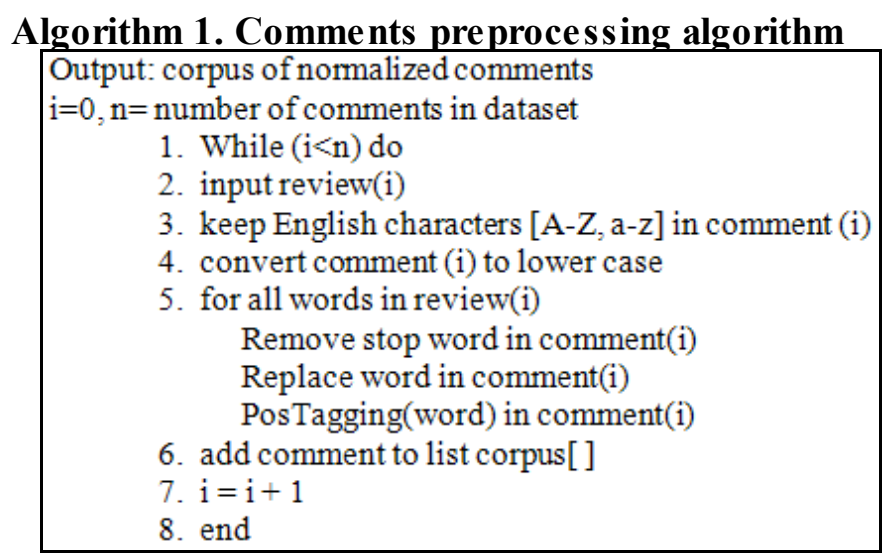


The preprocessing process proposed the comments text from parts, which decrease the efficiency of machine learning for sentiment analysis operation such as remove stop words, finding the PosTagging of words in each comment text, replace text and then saving the result refined text in corpus list database.

\section{SentiWordNet Score}

In table1 show SentiWordNet Score after the above calculation.

Table 1. SentiWordNet Score

\begin{tabular}{|c|c|c|}
\hline Comments by Shares & Score & Total Words \\
\hline Comment 1 & 0.36 & 68 \\
\hline Comment 2 & 0.29535 & 60 \\
\hline Comment 3 & 0.70894 & 20 \\
\hline
\end{tabular}

\subsection{Compute count for like, and share and weight link}

Compute count for like, and share and weight link by using Simplified PageRank Algorithm.

\subsubsection{Calculation of Improved Page Rank Algorithm}

Improved PageRank, a trust network inference algorithm, is used as the basic for generating predictive ratings personalized for each user. An Improved PageRank algorithm will propose based on time-weight and credit evaluation.

$\mathrm{PR}_{i}=(1-\mathrm{d})+\mathrm{d}\left[\sum_{j \in L i} \frac{P R_{j}}{o_{j}}\right]$, where $\mathrm{d}=$ Damping Factor (0.85), where PR = PageRank, Ij = Number of in links, $\mathrm{O}_{\mathrm{j}}=$ Number of out links, $\mathrm{I}_{\mathrm{k}}=$ total number of in links, $\mathrm{O}_{\mathrm{k}}=$ total number of out links.

In table 2. described the output result after the calculation show descending order for relevance trusted users.

Table. 2 Improved pageRank result

\begin{tabular}{|r|r|r|r|}
\hline Iteration & $\begin{array}{c}\text { Friend } \\
\text { A }\end{array}$ & $\begin{array}{c}\text { Friend } \\
\text { B }\end{array}$ & $\begin{array}{c}\text { Friend } \\
\text { C }\end{array}$ \\
\hline 1 & 0.800 & 1.200 & 1 \\
\hline 2 & 0.660 & 1.281 & 0.975 \\
\hline 3 & 0.694 & 1.274 & 0.986 \\
\hline--- & ---- & --- & --- \\
---- & ---- & -- \\
\hline 10 & 0.700 & 1.296 & 0.998 \\
\hline 11 & 0.701 & 1.296 & 0.999 \\
\hline 12 & 0.701 & 1.297 & 0.999 \\
\hline
\end{tabular}

$\mathrm{R}=\frac{\sum_{i f f} \mathrm{PR} f i \mathrm{~F} f i}{\mathrm{PR} f i}$, where $\mathrm{R}=$ recommended rating, $\mathrm{PR}_{f i}=$ total weighted by the trust value from to each user by shares post, $\mathrm{F}_{f i}=$ Score result from each comment by shares post.

\subsubsection{Simple result}

Table. 3 Simple result for trust calculation

\begin{tabular}{|c|c|c|c|}
\hline Friends & $\mathrm{PR}_{f i}$ & $\mathrm{~F}_{f i}$ & $\mathrm{PR}_{f i} \mathrm{~F}_{f i}$ \\
\hline A & 1.297 & 0.36000 & 0.46692 \\
\hline B & 0.701 & 0.29535 & 0.20704035 \\
\hline C & 0.999 & 0.70894 & 0.70823106 \\
\hline
\end{tabular}

$\mathrm{R}=\frac{1.297 * 3+0.701 * 5+0.999 * 4}{1.297+0.701+0.999}=1.38519141$ 
The final result calculates trust using above weighted result and score result and to output the trust decision making. This value becomes the "Trust Recommended".

\section{Conclusion and Future work}

In this paper is proposed inferring trust values from existing customers in online shopping. The benefit of the proposed approach is that it's not need direct ratings of customers. Accordingly, it can be used in any online shopping and social networks where some contextual information about the customer is available. Moreover, this system proposed to implement recommending applied on data from online shopping and how the results of sentiment analysis of textual comments. This evaluation can be used for evaluating customer sentiment regarding online shopping and it can also be applied to any relevant and sufficiently large data set containing sentiment data. Also, sentiment analysis success in predicting trust for customer based on comments in shares by post and also Improved PageRank Algorithm is easier to find the most trusted path in as minimum time more possible than other and at the time of trust calculation. In the future, customer's reviews is absolutely important and calculate more appropriate techniques based on this system for trust in online shopping.

\section{References}

[1] Amel Ziani, Nabiha Azizi, Didier Schwab, Monther Aldwairi, Nassira Chekkai,Djamel Zenakhra, Soraya Cheriguene, "Recommender System Through Sentiment Analysis", 13 Jan 2018.

[2] Au Yau Leung,Rex, "A StudyoftheTrust in On-line Shopping in HongKong: Differences between Scholars and Students", April 2005.

[3] Dimah Alahmadi and Xaio-Jun Zeng, "Improving Recommendation Using Trust and Sentiment Inference from OSNs", journal, Vol. 1, No. 1, June 2015.

[4] R. Lydia Priyadharsini, M. Lovelin Ponn Felciah, "Recommendation System in E-Commerce using Sentiment Analysis", journal, Volume 49 Number 7 July 2017

[5] Sonja Grabner-Krauter and Sofie Bitter, "Trust in online social network : A multifaced perspective", 2015

[6] DaireO’Dohery, Salim Jouili, Peter Van Roy, "Trust - Based Recommendation: an Empirical Analysis".

[7] Betsy Baby, Soumya Murali, "A Survey on Trust Based Recommendation Systems collaborative filter in trust Propagation, matrix factorization." 2016 Journal.

[8] Jiliang Tang, "Computing Distrust in Social Media", Approved February 2015.

[9] Justin Zhan, Xing Fang "A Computational Trust Framework for Social Computing”, paper IEEE 978-0-76954211-9 2010.

[10] Munmun Bhattacharya, , Kolkata Nashreen Nesa, "An Algorithm for Predicting Local Trust based on Trust Propagation in On line Social Networks", International Journal of Computer Applications (0975 - 8887) Volu me 156 - No 7, December 2016.

[11] Manasa S. M., Manjula S. H., Venugopal K. R., "Trust Aware System for Social Networks: A Comprehensive Survey", International Journal of Computer Applications (0975 - 8887) Volume 162 - No 5, March 2017

[12] Peter Hakansson, Hope Witmer, "Social Media and Trust - A Systematic Literature Review", Journal of business and economics, ISSN 2155-7950, USA, March 2015.

[13] Bo Yang, Yu Lei, Jiming Liu, Fellow, "Social Collaborative Filtering by Trust", 2017 IEEE.

[14] Darshana Karna, Ilsang Ko, We-Intention, Moral Trust and Self-Motivation on Accelerating Knowledge Sharing in Social Collaboration", 2015 IEEE.

[15] Wanita Sherchan, IBM Research- Australia, "A Survey of Trust in Social Networks",ACM Computing Surveys, Vol. 45, No. 4, Article 47, Publication date: August 2013.

[16] Wenpu Xing and Ali Ghorbani, "Weighted PageRank Algorithm".

[17] Xiao ming Zheng, Yan Wang, Mehmet A. Orgun, "BiNet: Trust Sub-network Extraction using Binary Ant Colony Algorithm in Contextual Social Networks" 2015 IEEE.

[18] Yao Ma, Hongwei Lu, Zaobin Gan, "An Improved Direct Trust Evaluation Algorithm for the Context-a ware Trust Model", 2013 IEEE. 